\title{
Cumplimiento en la notificación de casos probables de dengue en el Área Metropolitana Centro Occidente de Colombia en el año 2014
}

\author{
Germán Alberto Moreno Gomez ${ }^{1}$, Jhon Gerardo Moreno Gómez ${ }^{2}$ Ángela María Cabezas Restrepo ${ }^{3}$, Juan Esteban Múnera Benavides ${ }^{3}$, \\ Katherin Paola Ocampo Alzate ${ }^{3}$, Valentina Moreno Villegas ${ }^{3}$
}

\author{
Médico, Magister en Epidemiologia Clínica, Docente titular Universidad Tecnológica de Pereira; \\ Médico, Especialista en Gerencia de Servicios de Salud, Docente Universidad Tecnológica de Pereira \\ Estudiante de Medicina Universidad Tecnológica de Pereira \\ gamor@utp.edu.co, gamoreno2@gmail.com.
}

Fecha de recibido: Marzo 1 de 2016 Fecha de corrección: Marzo 13 de 2016 Fecha de aprobación: Marzo 16 de 2016

\begin{abstract}
Resumen
Objetivo: Cuantificar el cumplimiento de notificación de casos probables de dengue en el área Metropolitana Centro Occidente de Colombia, en el año 2014. Métodos: Esta investigación corresponde a un estudio observacional, descriptivo de corte transversal a partir de los registros de diagnósticos de dengue y sus diagnósticos diferenciales identificados en los Registros Individuales de Prestación de Servicios (RIPS) y el Sistema Nacional de Vigilancia en Salud Pública (SIVIGILA) del año 2014, de las Unidades Primarias Generadoras de Datos (UPGD) del Área Metropolitana Centro Occidente del Departamento de Risaralda. Se seleccionaron todos los registros de dengue (Censo) y se obtuvo una muestra de los diagnósticos diferenciales con un nivel de confianza del $95 \%$, una p de 0.5 y una precisión del 5\%. Posteriormente se calcularon porcentajes de cumplimiento en la notificación para dengue y para casos probables. Resultados: El 64\% de los pacientes con diagnósticos de dengue fueron notificados al SIVIGILA en el año 2014. El 10,7\% de los diagnósticos diferenciales cumplieron con el criterio de caso probable de dengue y el $12,9 \%$ de los casos probables de dengue fueron notificados al SIVIGILA en el año 2014. Discusión: Los resultados de este estudio son importantes porque brindan información sobre el cumplimiento de la notificación obligatoria de los casos de dengue al Sistema de Vigilancia Epidemiológica del dengue de las UPGD públicas del Área Metropolitana Centro Occidente del Departamento de Risaralda, evidenciando un incumplimiento en la notificación. Además, al comparar los resultados con otros estudios realizados tanto a nivel internacional (Costa Rica, Brasil y Chile) y a nivel nacional (Santander y Valle) se observan problemas similares de notificación en los Sistemas de Vigilancia Epidemiológica respectivos.
\end{abstract}

Palabras Clave: Dengue; Vigilancia epidemiológica, Omisiones de registro
Compliance in reporting probable cases of dengue in the Colombian West Metropolitan Area in 2014

\begin{abstract}
Objectives: Quantifies the compliance of notification concerning to probable cases of dengue in the West Metropolitan Area in 2014. Methods: This research corresponds to an observational cross-sectional study that has as populations the sets of records about dengue diagnoses and differential diagnoses identified in the individual records of provide services (RIPS) and the surveillance system in public health (SIVIGILA) in 2014 of the elementary generating data units (UPGD) in the West Metropolitan Area of the department of Risaralda. All the records of dengue were selected (census) and a sample of the differential diagnoses was obtained with a confidence level of the $95 \%$, a $p=0,5$ and an accuracy of the $5 \%$. Subsequently compliance rates were calculated for dengue and differential diagnoses. Results: $64 \%$ of dengue diagnosis's patients were reported to SIVIGILA in 2014. The $10.7 \%$ of the differential diagnoses fulfill criteria for probable case of dengue and $12,9 \%$ of probable cases of dengue were reported to SIVIGILA in 2014. Discussion: The results of this research are important as they provide useful information about the epidemiological surveillance system in dengue for the public UPGD in the West Metropolitan Area of the department of Risaralda, where a deficit is evident in the notification. Also to compare the results with other international (Costa Rica, Brazil and Chile) and national (Santander and Valle) studies, similar problems are observed in the respective Epidemiological Surveillance Systems
\end{abstract}

Key Words: dengue; mandatory reporting; dengue virus; under-registration; epidemiological surveillance.

\section{Introducción}

El dengue es un enfermedad febril, aguda de etiología viral, transmitida por la hembra de los mosquitos del género Aedes aegypti o Aedes albopictus en zonas tropicales y subtropicales que se encuentran ubicadas hasta $1800 \mathrm{msnm}\left({ }^{1}\right)$. Desde el punto de vista clínico existen dos tipos de dengue: el dengue clásico y el dengue grave, siendo el dengue grave una complicación de la enfermedad $\left(^{2}\right)$.

A nivel mundial el 40\% de la población se encuentra en riesgo de infección; anualmente se presentan aproximadamente 50 millones de infecciones $\left({ }^{3}\right)$. En Colombia el 50,4\% de la población vive en regiones susceptibles de contraer la enfermedad y es considerado como un problema de interés en salud pública y está incluido en el Sistema de Vigilancia Epidemiológica colombiano, SIVIGILA, como una enfermedad de notificación obligatoria $\left({ }^{4}\right)$. Para la semana epidemiológica 53 de 2014 se habían notificado en Colombia, 110.473 casos de dengue, de los cuales el 2,5\% (2777) fueron clasificados como dengue grave. Del total fueron confirmados 46139 (43\%), la tasa de letalidad fue del 3,92\% (109 casos), dos de las muertes en Risaralda $\left({ }^{5}\right)$

Existe evidencia en diferentes estudios, realizados en Colombia y otros países Latinoamericanos, que sugiere problemas en la notificación entre los casos probables y los registrados en el sistema de vigilancia, lo que puede estar generando un subregistro $\left(^{6}\right)\left({ }^{7}\right)\left({ }^{8}\right)\left({ }^{9}\right)$. Zea y Osorio encontraron en un municipio de Colombia que un escaso número de funcionarios eran responsables del sistema de notificación de casos, con poca articulación de las actividades y sin la capacitación suficiente. Por otra parte del 91,3\% de las UPGD que enviaron los reportes, solo una reportó durante todas las semanas epidemiológicas y siete hicieron el reporte en medios físicos por no contar con la herramienta informática (SIVIGILA) $\left({ }^{10}\right)$. 
Sáenz E y colaboradores en 2001 evaluaron el Sistema de Vigilancia Epidemiológica del dengue en Costa Rica utilizando como indicador la aplicación de la definición de caso probable. Se estudiaron 377 casos de los cuales encontraron 114 (30\%) casos probables, y de estos, 36 casos fueron confirmados; 263 de los casos estudiados no cumplieron con la definición de caso probable, y el 36\% de los pacientes con los criterios establecidos no se notificaron al sistema. Los resultados demostraron que el sistema basado en la vigilancia clínica de los casos probables no es sensible (7).

Romero Vega y colaboradores, en el año 2014, revisaron 13.873 casos procedentes de 45 instituciones de 11 departamentos de Colombia, de estos se obtuvo una muestra de 4.094 pacientes con Síndrome Febril Agudo (SFA) y 265 con diagnósticos de egreso de dengue; al aplicar los criterios de la OMS, 2.977 se consideraron sin dengue y 1.382 pacientes compatibles con dengue, de los cuales 210 fueron notificados al SIVIGILA, para un cumplimiento en la notificación del $15,2 \%\left({ }^{11}\right)$

Todo lo anterior refuerza la preocupación que existe en la Secretaria de Salud Departamental de Risaralda por la poca congruencia que existe entre los casos de dengue registrados en los RIPS y los notificados en el SIVIGILA; el número de casos confirmados de dengue y dengue grave en el Área Metropolitana Centro Occidente de Risaralda fueron 587, en el departamento de Risaralda fueron 651, para Quindío fueron 1.731 y para Caldas 800 en el año 2014. Estas diferencias con Risaralda, se podrían explicar por un sub-registro ya que estos departamentos cuentan con condiciones económicas, sociales y geográficas similares $\left({ }^{12}\right)$.

Esta investigación pretende evaluar un componente del Sistema de Vigilancia Epidemiológica, específicamente la notificación y registro de casos probables de dengue en las Unidades Primarias Generadoras de Datos (UPGD) del área metropolitana Centro Occidente el año 2014, lo que permitirá establecer si la notificación del dengue se hace de acuerdo a lo establecido en el Protocolo de Vigilancia del Instituto Nacional de Salud, (2) lo que se justifica por las implicaciones de tipo económico, social y de salud pública que tiene la enfermedad $\left({ }^{13}\right)\left({ }^{14}\right)$ $\left({ }^{15}\right)$.

\section{Materiales y Métodos}

Esta investigación corresponde a un estudio observacional descriptivo de corte transversal. Se solicitaron a la Secretaria de Salud Departamental, los Registros Individuales de Prestación de Servicios (RIPS) de las UPGD públicas del área Metropolitana Centro Occidente, del año 2014.

Se identificaron los registros de dengue en los RIPS (cuadro 1), con los códigos A90X y A91X del CIE10 (código internacional de enfermedades decima revisión) $\left({ }^{16}\right)$, y se compararon con la base de datos SIVIGILA.

Con base en los códigos del CIE 10 que corresponden a los diagnósticos diferenciales de dengue, se seleccionaron los registros de los RIPS y se calculó la muestra con un nivel confianza del 95\%, una p 0.5 , y una precisión del 5\%. Previa capacitación en los criterios diagnósticos establecidos en el protocolo de vigilancia, se revisaron las historias clínicas de los registros seleccionados, se identificaron los casos probables de dengue y se verificó su notificación en el SIVIGILA (cuadro 2) $\left({ }^{17}\right)$
Se calculó el porcentaje de cumplimiento de la notificación de los casos de dengue encontrados en los RIPS con respecto al SIVIGILA, de los diagnósticos diferenciales de dengue que cumplieron con el criterio de caso probable con respecto al SIVIGILA y un porcentaje de cumplimiento global. Según la resolución 8430 de 1993 se trató de una investigación sin riesgo y fue avalada por el Comité de bioética de la Universidad Tecnológica de Pereira

\section{Resultados}

Se compilaron 892.697 registros de los RIPS del año 2014, incluyendo consulta externa, urgencias y hospitalización de los cuales 422.215 $(47,3 \%)$ correspondieron a la UPGD1, $72.817(8,15 \%)$ a la UPGD2 $247.602(27,73 \%)$ a la UPGD3, $118.452(13,26 \%)$ a la UPGD4, y $31.611(3,54 \%)$ a la UPGD5.

\section{Casos de dengue:}

Cuadro 1: Pacientes con diagnóstico de dengue por UPGD en el Área Metropolitana Centro Occidente año 2014.

\begin{tabular}{rcccc}
\hline Entidad & $\begin{array}{c}\text { Dx } \\
\text { Dengue }\end{array}$ & Porcentaje & $\begin{array}{c}\text { Casos } \\
\text { notificados }\end{array}$ & \% notificación \\
\hline UPGD1 & 254 & $46,27 \%$ & 172 & $67,7 \%$ \\
UPGD2 & 135 & $24,59 \%$ & 83 & $61,48 \%$ \\
UPGD3 & 86 & $15,66 \%$ & 41 & $47,77 \%$ \\
UPGD4 & 74 & $13,48 \%$ & 56 & $75,67 \%$ \\
UPGD5 & 0 & $0,00 \%$ & 0 & $0,00 \%$ \\
Total & 549 & $100 \%$ & 352 & $64 \%$ \\
\hline
\end{tabular}

Del total de registros, se identificaron 549 pacientes con diagnóstico de dengue, el mayor porcentaje (46,27\%) provino de la UPGD1 (Cuadro 1). La UPGD2 es la institución que tiene la tasa más alta de registro de dengue por cada 100.000 registros de RIPS con 185, seguido de la UPGD4 con 62x100.000 y la UPGD1 con 60x100.000 y la de menor reporte la UPGD3 con 34x100.000 registros.

De los 549 pacientes con diagnóstico de dengue se notificaron al SIVIGILA 352, con un porcentaje de cumplimiento en la notificación del 64\%. UPGD4 es la institución que presenta el porcentaje más alto de notificación $(75,67 \%)$ y la UPGD3 es la institución con el menor porcentaje de notificación $(47,77 \%)$.

\section{Casos probables de dengue:}

Cuadro 2: Registro de diagnósticos diferenciales de dengue por UPGD y por servicio Área Metropolitana Centro Occidente año 2014.

\begin{tabular}{cccccc}
\hline Entidad & Urgencias & $\begin{array}{c}\text { Consulta } \\
\text { Externa }\end{array}$ & Hospitalización & Total & $\%$ \\
\hline UPGD1 & 80 & 1655 & 455 & 2190 & $48,1 \%$ \\
UPGD2 & 7 & 355 & 490 & 852 & $18,7 \%$ \\
UPGD3 & 34 & 541 & 176 & 751 & $16,5 \%$ \\
UPGD4 & 75 & 633 & 40 & 748 & $16,44 \%$ \\
UPGD5 & 3 & 2 & 3 & 8 & $0,1 \%$ \\
Total & 199 & 3186 & 1164 & 4549 & \\
Porcentaje & $4,37 \%$ & $70 \%$ & $25,6 \%$ & & $\mathbf{1 0 0} \%$ \\
\hline
\end{tabular}


Del total de registros de la base de datos, se identificaron 4.549 diagnósticos diferenciales de dengue, el mayor porcentaje $(48,1 \%)$ procedían de la UPGD1 (Cuadro 2). Los servicios de urgencias son los que mayor número de diagnósticos diferenciales reportan con una tasa de 15,4x1.000 consultas, seguido de consulta externa con $3,8 \times 1.000$ consultas y hospitalización con $2,7 \times 1.000$ egresos.

Con base en la muestra calculada, se revisaron 291 historias clínicas de pacientes con diagnósticos diferenciales de dengue, correspondientes a las UPGD así: UPGD1 (132), UPGD2 (62), UPGD4 (52) y UPGD3 (45). El $10,7 \%$ (31) de las historias revisadas, cumplieron con los criterios de caso probable de dengue y de estas el 12,9\% (4) se notificaron al SIVIGILA.

Si se tienen en cuenta los casos de dengue (549) y los casos probables (31) de dengue el porcentaje de cumplimiento global de la notificación en el SIVIGILA fue del $61,3 \%$.

\section{Discusión}

Con el objetivo de calcular el porcentaje de cumplimiento de notificación de los casos de dengue y los casos probables de dengue de las UPGD públicas del Área Metropolitana Centro Occidente en el año 2014, se analizaron los Registros Individuales de Prestación de Servicios (RIPS) que correspondían a los diagnósticos de dengue y los diagnósticos diferenciales que cumplían con el criterio de caso probable; se obtuvo un porcentaje de cumplimiento global de notificación en el SIVIGILA del 61\%, un porcentaje de cumplimiento del $64 \%$ para los casos dengue y $12,9 \%$ para los diagnósticos diferenciales, lo que confirma el sub-registro en la notificación en las UPGD estudiadas.

Al comparar los resultados obtenidos con otros estudios, Saenz E y colaboradores en 2001, evaluaron el Sistema de Vigilancia Epidemiológica del dengue en Costa Rica y encontraron que el 36\% de los pacientes con los criterios de caso probable no se notificaron al sistema (11), porcentaje igual al obtenido en este estudio $(38,7 \%)$. Un porcentaje similar obtuvieron Heliosa Duarte y Elisabeth Barboza, al evaluar la calidad de la información notificada en el Sistema de Vigilancia para la detección de casos de dengue en Brasil, encontrando un subregistro del $37 \%$ de casos que cumplían con los criterios establecidos $\left({ }^{18}\right)$. Canls, González y Figueroa, en el año 2012, estudiaron la dinámica del dengue en la isla de Pascua, Chile, durante las epidemias del 2002-2011 y encontraron que el número de casos esperados fue mayor al número de casos notificados, lo que sugiere un subregistro $\left({ }^{19}\right)$. En Colombia, Romero-Vega y colaboradores, en el año 2014, encontraron para 11 departamentos un porcentaje mayor de subregistro con un valor del $85 \%$ (12). Castañeda y colaboradores en conjunto con la Secretaría Departamental de Salud de Santander, en el año 2010, revisaron 4.774 RIPS, donde encontraron 67 registros con diagnóstico diferencial compatible con dengue, de los cuales 16 casos, del nivel municipal, cumplían los criterios de caso probable establecidos en el Protocolo, de los cuales 9 no se reportaron al SIVIGILA, presentándose un subregistro del $56 \%\left({ }^{20}\right)$.

Los datos anteriores sugieren problemas de notificación en los Sistemas de Vigilancia Epidemiológica en algunos países de la región; entre las posibles causas que han sido descritas están: el mal diligenciamiento de la ficha de notificación (6), desconocimiento o falta de capacitación para el cumplimiento del Protocolo de Vigilancia en Salud Pública de Dengue por parte de los profesionales de la salud; desinterés por la notificación de casos probables debido a la documentación adicional que debe llenar el médico; diversidad de diagnósticos diferenciales que dificulta su identificación; falta de recursos para la notificación (software SIVIGILA) y la poca vigilancia para el cumplimiento de la norma (7) (10).

Los resultados de este estudio son importantes en la medida en que brindan información útil sobre el Sistema de Vigilancia Epidemiológica del dengue para las UPGD públicas del Área Metropolitana Centro Occidente del Departamento de Risaralda; no se incluyeron los registros de las IPS privadas ni de los otros municipios del Departamento. Se evidenció un insuficiente registro de información en las historias clínicas, lo que puede estar generando un subregistro adicional de casos probables.

De acuerdo a lo establecido por el Instituto Nacional de Salud el cumplimiento de notificación esperado debe ser del 84\% (21); es evidente que las UPGD públicas del Área Metropolitana no están cumpliendo con la notificación, lo cual es preocupante por las implicaciones que tiene en la salud pública, lo que amerita continuar con una línea de investigación que establezca el porcentaje de cumplimiento de la notificación en todas las UPGD públicas y privadas del Departamento, así como las causas de no notificación para implementar estrategias que conduzcan al fortalecimiento del Sistema de Vigilancia Epidemiológica.

\section{Conflicto de Interés}

Los autores declaramos que no existe conflicto de interés en la elaboración de este artículo.

\section{Agradecimientos}

Agradecemos a la Secretaría Departamental de Salud de Risaralda y a las UPGD Públicas por permitir el desarrollo del proyecto al facilitar la información para la recolección de datos.

\section{Referencias}

1 Organización Mundial de la Salud. Temas de Salud. Dengue. Disponible en: www.who.int/topics/dengue/es/. Visitado 28/10/2014.

2 República de Colombia. Ministerio de la Protección Social, Instituto Nacional de Salud. Vigilancia y análisis del riesgo en salud pública. Protocolo de vigilancia en salud pública de dengue. Bogotá Junio del 2014

3 Organización Panamericana de la Salud. Organización Mundial de la Salud. Dengue: Guías para el diagnóstico, tratamiento, prevención y control la Paz. Bolivia. OPS/OMS

4 República de Colombia. Ministerio de la Protección Social. Decreto 3518. Octubre 2006.

5 República de Colombia. Instituto nacional de Salud. Boletín epidemiológico semana 53 de 2014.

6 Universidad Federal Fluminense, evaluación del completamiento de los registros de dengue estudio exploratorio de las notificaciones compulsorias, Fundación Municipal de la Salud de Niterói, Fluminense diciembre 2012.

7 Saenz E, Maranda L, González L. Adecuación de la definición de Casos Sospechosos de Dengue, basado en la Asociación de Síntomas y Signos según los Registros Médicos. Rev: Costarric. Cienc. Med Vol.22 No. 3-4 San José. Dic 2001. 
8 Cassia Magri, Christiane. Análisis sobre la notificación de casos de dengue en la red de salud ivirgarzama gestiones. Bolivia 20062007.

9 Castañeda C, et al. Burden of Disease and Economic Impact of Dengue and Severe Dengue in Colombia, 2011. Value in Health Regional Issues 1 (2), 123 -128.

10 Zea D, Osorio L. Situación del Sistema de Vigilancia de Casos de Dengue en un Municipio de Colombia. Rev: Salud Pública. 13 (5): 785-795, 2011.

11 Romero-Vega L, Pacheco O, de la Hoz-Restrepo F, Díaz-Quijano FA. Evaluación de la notificación del dengue durante una epidemia. Colombia. Revista de Saúde Pública. 2014;48(6):899905. doi:10.1590/S0034-8910.2014048005321.

12 República de Colombia. Instituto Nacional de Salud. Sivigila. Vigilancia rutinaria 2014.Disponible en http://www.ins.gov. co/lineas-de-accion/subdireccion-vigilancia/sivigila/paginas/ vigilancia-rutinaria.aspx. Consultado 13-03-2016.

13 Castro R. Costos del dengue en Colombia (2010-2012). Impacto en salud pública. Universidad de los Andes. Disponible en: http:// www.paho.org/hq/index.php?option $=$ com_docman\&task $=$ doc_ view\&gid=25900\&Itemid=. Visitado: 28/10/2014

14 Universidad Industrial de Santander. Centro de investigaciones epidemiológicas. Dificultad para el diagnóstico clínico temprano del dengue en un área endémica y su impacto sobre el manejo medico inicial. Colombia, Bucaramanga, Marzo 2006.

15 Fossaert H, Llopis A, Tigre C. Sistemas de Vigilancia Epidemiológica. Boletín de la Oficina Sanitaria Panamericana. OPS. Junio 1974.

16 República de Colombia. Ministerio de Salud y de la Protección Social. Tabla de clasificación estadística internacional de enfermedades y problemas relacionados con la salud, Décima revisión (CIE-10) para el Registro Individual de Prestación de Servicios (RIPS) con restricciones de sexo, edad y códigos que nos son afección principal. 2008.

17 Organización Panamericana de la Salud, Oficina Sanitaria Panamericana y Oficina Regional de la Organización Mundial de la Salud. Módulos de principios de Epidemiología para el control de enfermedades (MOPECE). Segunda edición. Washington D.C.: OPS, ( ) 2002, 49 p. (Serie PALTEX Nº para Técnicos Medios y Auxiliares $\left.\mathrm{N}^{\circ} 24\right)$.

18 Duarte Heloisa Helena Pelluci, França Elisabeth Barboza. Qualidade dos dados da vigilância epidemiológica da dengue em Belo Horizonte, MG. Rev. Saúde Pública [Internet]. 2006 Feb [cited 2015 June 14]; 40(1): 134-142. Available from: http:// www.scielo.br/scielo.php?script=sci_arttext\&pid $=$ S0034$89102006000100021 \& \operatorname{lng}=$ en http://dx.doi.org/10.1590/S003489102006000100021

19 Canals, Mauricio; González, Christian; Canals, Andrea; Figueroa, Daniela. Dinámica epidemiológica del dengue en Isla de Pascua. Rev Chilena Infectol; 29(4): 388-394. Agosto. 2012.

20 Secretaría de Salud de Santander, Castañeda, Segura, Ramírez, Conocimientos, actitudes y prácticas comunitarias en un brote de Dengue en un municipio de Colombia, Bucaramanga, Colombia, 2010.
21 Instituto Nacional de Salud. Dirección de Vigilancia y Análisis del Riesgo en Salud Publica. Boletín Epidemiológico Semanal, 2014. 